\title{
Incidencia de las estrategias metodológicas activas en el aprendizaje significativo del idioma inglés
}

\section{Incidence of active methodological strategies in the meaningful learning of the English language}

\section{Incidencia de las estrategias metodológicas activas en el aprendizaje significativo del idioma inglés}

Edison S. Sanguña-Loachamin ${ }^{\mathrm{I}}$ essanguna@uce.edu.ec

\author{
Boris E. Vasconez-Torres ${ }^{\text {II }}$ \\ bevasconez@uce.edu.ec \\ Amilcar O. Gonzalez-Diaz ${ }^{\text {III }}$ \\ aogonzalez@uce.edu.ec \\ Gabriela M. Moso-Mena IV \\ gmmoso@uce.edu.ec
}

Correspondencia: essanguna@uce.edu.ec

I. Magister en Docencia Universitaria y Administración Educativa; Especialista en Diseño Curricular por Competencias; Licenciado en Ciencias de la Educación mención: Plurilingue, Docente de la Universidad Central del Ecuador, Quito, Ecuador.

II. Magister en Educación; Especialista en Currículo y Didáctica; Diploma Superior en Investigación Socioeducativa; Ingeniero en Diseño Gráfico Empresarial; Licenciado en Lingüistica Aplicada al Idioma Ingles; Licenciado en Ciencias de la Educación mención Diseño y Dibujo, Docente de la Universidad Central del Ecuador, Quito, Ecuador.

III. Magister en Linguistica y Didáctica de la Enseñanza de Idiomas Extranjeros; Diploma Superior de la Enseñanza de Inglés como Segunda Lengua; Licenciado en Ciencias de la Educación mención Ingles; Técnico Superior en Turismo, Docente de la Universidad Central del Ecuador, Quito, Ecuador.

IV. Magister en Educación Superior y Equidad de Género; Licenciada en Ciencias de la Educación mención Plurilingue, Docente de la Universidad Central del Ecuador, Quito, Ecuador. 


\section{Resumen}

El trabajo de investigación denominado: "Incidencia de las estrategias metodológicas activas en el aprendizaje significativo del idioma inglés" se desarrolló en base a la realidad existente en la sección primaria de la Unidad Educativa Particular "Cristiano Verbo" de la ciudad de Quito, frente a la problemática existente de insuficiente aplicación de metodologías activas para el enseñanza del idioma inglés que fue evidenciado por medio de una encuesta aplicada a los estudiantes, profesores y autoridades de la institución mencionada, se planteó una alternativa de solución que se denominó "Guía de métodos alternativos para la enseñanza del idioma inglés" propuesta que ofrece innovadores métodos de enseñanza especializados para la enseñanza de idiomas extranjeros, ello abre un abanico de alternativas para los docentes de inglés, puesto que cada maestro tendrá a su disposición una variedad de actividades para su buen desempeño docente, también, dicho trabajo propone mayores oportunidades a los estudiantes, motivándoles al aprendizaje del inglés de una manera práctica y en un ambiente natural de la misma forma como adquirieron su lengua materna, mejorando de esta forma su rendimiento académico, por ende alcanzando aprendizajes significativos del idioma inglés.

Palabras claves: Estrategias metodológicas; métodos de enseñanza; técnicas de enseñanza; didáctica; aprendizaje significativo; psicología del aprendizaje; procesos de aprendizaje; rendimiento académico; habilidades; destrezas. 


\section{Abstract}

The research work entitled "Incidence of active methodological strategies in the meaningful learning of the English language" was developed based on the reality existing in the primary section of the "Christian Verbo" Educational Unit of the city of Quito. the existing problem of insufficient application of active methodologies for English language teaching was evidenced by a survey applied to the students, teachers and authorities of the mentioned institution, an alternative solution was proposed that was called "Guide of alternative methods for the teaching of the English language "proposal that offers innovative specialized teaching methods for the teaching of foreign languages, this opens a range of alternatives for English teachers, since each teacher will have at his disposal a variety of activities for his good performance teacher too $n$, this work proposes greater opportunities for students, motivating them to learn English in a practical way and in a natural environment in the same way as they acquired their mother tongue, thus improving their academic performance, thus achieving meaningful language learning English.

Key words: Methodological strategies; teaching methods; teaching techniques; didactics; significant learning; Psychology of Learning; learning processes; academic performance; abilities; abilities. 


\section{Introducción.}

En el presente siglo y en todo el mundo se habla mucho de ciencia y tecnología, los mismos que brindan un grandioso aporte al desarrollo de una sociedad cada vez más exigente y globalizada, bajo este nuevo estilo de vida, cada vez se hace más imprescindible el aprendizaje del idioma inglés, esto es por la utilización que se le da en diferentes ámbitos de la vida diaria, por solo ejemplo: los automóviles, las computadoras, los electrodomésticos y todo artefacto eléctrico y electrónico trae consigo guías para el usuario y es una necesidad que el texto esté redactado en inglés y luego en los idiomas de acuerdo al país de destino, los negocios, la información, los intercambios interculturales entre países de diferentes lenguas se los realiza en inglés; en la actualidad para el profesional es importante añadir a su hoja de vida por lo menos la suficiencia del idioma inglés para tener mayores oportunidades de trabajo y mejores ingresos económicos, otro campo de utilidad es el turismo; si una persona viaja a un país extranjero y no habla el idioma local, fácilmente podrá desenvolverse si habla inglés; por estas y otras razones el idioma inglés es considerado como un idioma universal, un idioma que todas las personas deben hablarlo como un segundo idioma. En lo concerniente al ámbito educativo el idioma inglés también evidencia grandes expectativas, desde los primeros años de educación básica hasta los estudios de nivel superior las instituciones educativas ponen énfasis en la enseñanza del inglés, en la actualidad dominar el idioma permite al nuevo profesional ampliar sus horizontes en el ámbito laboral mejorando su calidad de vida y contribuyendo al desarrollo de la sociedad. (Duarte de Kendler, 2007) (Brown, 2000)

A de decir de (Richards \& Rodgers , 2001) “La historia de la enseñanza del idioma inglés ha sido caracterizada por una búsqueda de maneras más efectivas de enseñar un idioma, ya sea como segunda lengua, o como una lengua extranjera.” (p vii) 
Edison S. Sanguña-Loachamin; Boris E. Vasconez-Torres; Amilcar O. Gonzalez-Diaz; Gabriela M. Moso-Mena

En el presente siglo debe ser primordial la creación de nuevas estrategias metodológicas que ayuden a los estudiantes a la adquisición y dominio del idioma inglés, el mismo que será una herramienta para que puedan enfrentarse al reto de mantener una comunicación entre todos los países que no hablan la misma lengua pero que tienen al inglés como segundo idioma, para ello, es necesario innovar los actuales modelos educativos considerando la creación de espacios apropiados y oportunidades suficientes para desarrollar un aprendizaje efectivo del idioma inglés. (González, 2000) (Lightbown \& Spada, 1996)

El presente estudio tiene como objetivo profundizar las estrategias metodológicas activas en el aprendizaje significativo del idioma inglés, en los estudiantes de $5^{\circ}$ a $7^{\circ}$ año de la sección primaria de la Unidad Educativa Particular "Cristiano Verbo" de la ciudad de Quito, Año Lectivo $2009-2010$.

\section{Materiales y métodos.}

Desde el paradigma crítico-propositivo, la investigación tiene un enfoque de carácter cuanticualitativo. Cuantitativo porque los resultados de la investigación de campo serán sometidos a cálculos matemáticos- estadísticos. Cualitativo porque los resultados estadísticos serán procesados con análisis e interpretaciones críticas con el apoyo del marco teórico.

\section{Modalidades de Investigación}

\section{Bibliográfica Documentada}

El trabajo de investigación tendrá modalidad bibliográfica porque se recurrirá a fuentes de información secundaria obtenida en libros, revistas, publicaciones, internet, fotos, otros. Documental porque de ser necesario se dispondrá de fuentes primarias a través de actas, registros, documentos oficiales, otros. 
De campo

Porque el investigador acudirá a recabar información en el lugar en donde se producen los acontecimientos.

Proyecto Factible o de Intervención Social

Porque el investigador no se conforma con las conclusiones de la investigación, sino que además planteará una propuesta de solución al problema investigado.

\section{Población}

\begin{tabular}{|l|c|c|}
\hline POBLACIONES & CANTIDAD & PORCENTAJE \\
\hline Autoridades & 4 & $4 \%$ \\
\hline Docentes & 5 & $5 \%$ \\
& & \\
\hline Estudiantes & 95 & $91 \%$ \\
& & \\
\hline \multicolumn{1}{|c|}{ TOTAL } & $\mathbf{1 0 4}$ & $\mathbf{1 0 0} \%$ \\
\hline
\end{tabular}

\section{Técnicas e Instrumentos}

\section{Encuesta}

En la investigación se aplicó encuestas dirigidas a las autoridades del plantel, los docentes del área de inglés y a los estudiantes de $5^{\circ}$ a $7^{\circ}$ años de la sección primaria, basadas en un cuestionario elaborado con preguntas cerradas, enfocadas a recabar orientación sobre las variables de estudio. 


\section{Validez y Confiabilidad}

La validez de los instrumentos fue dada por la técnica "Juicio de expertos"; mientras que la confiabilidad se la hizo a través de la aplicación de una prueba piloto a una pequeña población, lo que sirvió para detectar errores y corregirlos a tiempo antes de su aplicación definitiva.

\section{Resultados.}

\begin{tabular}{|c|c|c|}
\hline OPCIONES & FRECUENCIA & PORCENTAJE \\
\hline SI & 26 & $27,37 \%$ \\
\hline NO & 69 & $72,63 \%$ \\
\hline TOTAL & 95 & $100 \%$ \\
\hline
\end{tabular}

Cuadro $N^{\circ} 1 .-$ ¿Le gusta aprender el idioma inglés?

De los 95 encuestados el 27,37\% respondieron que sí les gusta aprender el idioma inglés el $72,63 \%$ respondió que no le gusta, de lo que se desprende que el aprendizaje del idioma inglés no es atractivo para los estudiantes de $5^{\circ}$ a $7^{\circ}$ de básica.

\begin{tabular}{|c|c|c|}
\hline OPCIONES & FRECUENCIA & PORCENTAJE \\
\hline SI & 31 & $32,63 \%$ \\
\hline NO & 64 & $67,37 \%$ \\
\hline TOTAL & 95 & $100,00 \%$ \\
\hline
\end{tabular}

\section{Cuadro $N^{\circ} 2 .-$ ¿Las clases de inglés son interesantes?}

De los 95 estudiantes encuestados el 32,63\% manifiesta que las clases de inglés son interesantes, mientras que el $67,37 \%$ dice que no; por lo tanto las clases de inglés no se muestran interesantes para los estudiantes, ello indica que se deben aplicar nuevos métodos y técnicas de enseñanza para un mejor aprendizaje del idioma inglés. 


\begin{tabular}{|l|c|c|}
\hline \multicolumn{1}{|c|}{ OPCIONES } & FRECUENCIA & PORCENTAJE \\
\hline SI & 7 & $7,37 \%$ \\
\hline NO & 88 & $92,63 \%$ \\
\hline TOTAL & 95 & $100,00 \%$ \\
\hline
\end{tabular}

Cuadro $N^{\circ} 3 .-~ ¿ L o s$ profesores de inglés utilizan concursos, juegos en sus clases?

De los 95 estudiantes encuestados el 7,37\% menciona que los profesores sí realizan juegos para el aprendizaje del inglés, mientras que el 92,63\% dice que no; de modo que las clases no son atractivas para los estudiantes, ya que no realizan actividades lúdicas, las mismas que resultan ser motivadoras para el aprendizaje.

\begin{tabular}{|l|c|c|}
\hline \multicolumn{1}{|c|}{ OPCIONES } & FRECUENCIA & PORCENTAJE \\
\hline SI & 51 & $53,68 \%$ \\
\hline NO & 44 & $46,32 \%$ \\
\hline TOTAL & 95 & $100,00 \%$ \\
\hline
\end{tabular}

\section{Cuadro $N^{\circ} 4 .-$ ¿Los profesores trabajan con proyectos de aula en las clases de inglés?}

De los 95 encuestados el 53,68\% dice que los profesores sí trabajan con proyectos en sus clases y el 46,32\% manifiestan que no lo hacen; de lo que se deduce que urge la creación de proyectos de aula los cuales sirven como apoyo para la práctica del idioma dentro de las habilidades básicas de escuchar, hablar, leer y escribir en inglés. 
Edison S. Sanguña-Loachamin; Boris E. Vasconez-Torres; Amilcar O. Gonzalez-Diaz; Gabriela M. Moso-Mena

\begin{tabular}{|l|c|c|}
\hline \multicolumn{1}{|c|}{ OPCIONES } & FRECUENCIA & PORCENTAJE \\
\hline SI & 54 & 56,84 \\
\hline NO & 41 & 43,16 \\
\hline TOTAL & 95 & 100,00 \\
\hline
\end{tabular}

Cuadro $N^{\circ}$ 5.- ¿Aprenden inglés a través de canciones?

De los 95 estudiantes que realizaron la encuesta el 56,84\% dice que sí aprenden inglés a través de canciones y el 43,16\% menciona que no; deduciéndose que se deben reforzar los conocimientos con la ayuda de canciones en inglés, los mismos que aportan una gran cantidad de vocabulario y refuerza la repetición y posterior memorización de palabras y frases, y expresiones idiomáticas.

\begin{tabular}{|l|c|c|}
\hline \multicolumn{1}{|c|}{ OPCIONES } & FRECUENCIA & PORCENTAJE \\
\hline SI & 29 & $30,53 \%$ \\
\hline NO & 66 & $69,47 \%$ \\
\hline TOTAL & 95 & $100 \%$ \\
\hline
\end{tabular}

\section{Cuadro $N^{\circ}$ 6.- ¿Practican el inglés a través de diálogos?}

De los 95 estudiantes que realizaron la encuesta el 30,53\% dice que sí practican el inglés a través de diálogos y el 69,47\% menciona que no; lo que demuestra que los estudiantes no practican el idioma de una forma natural imitando, creando, o completando conversaciones relacionadas a la temática que se trate en las clases, dependiendo de la edad y el nivel de conocimientos de los estudiantes. 


\begin{tabular}{|l|c|c|}
\hline \multicolumn{1}{|c|}{ OPCIONES } & FRECUENCIA & PORCENTAJE \\
\hline SI & 23 & $24,21 \%$ \\
\hline NO & 72 & $75,79 \%$ \\
\hline TOTAL & 95 & $100 \%$ \\
\hline
\end{tabular}

Cuadro $N^{\circ}$ 7.- ¿Miran películas en inglés?

De los 95 estudiantes que realizaron la encuesta el 24,21\% dice que sí miran películas en inglés y el 75,79\% menciona que no; de lo que se desprende que urge implementar actividades audiovisuales como videos y películas los cuales resultan ser motivadores para los estudiantes y sirven como material de refuerzo para complementar su aprendizaje.

\begin{tabular}{|l|c|c|}
\hline \multicolumn{1}{|c|}{ OPCIONES } & FRECUENCIA & PORCENTAJE \\
\hline SI & 33 & $34,74 \%$ \\
\hline NO & 62 & $65,26 \%$ \\
\hline TOTAL & 95 & $100,00 \%$ \\
\hline
\end{tabular}

Caudro $N^{\circ}$ 8.- ¿Entienden las instrucciones en inglés que le da su maestro (a) para realizar actividades en clase?

De los 95 estudiantes que realizaron la encuesta el $34,74 \%$ dice que sí entienden las instrucciones dadas por el profesor y el $65,26 \%$ menciona que no entienden; de lo que se interpreta que los estudiantes deben realizar actividades orales de repetición a través de pictogramas lo que facilita el reconocimiento de los significados sin necesidad de una explicación.

\begin{tabular}{|l|c|c|}
\hline \multicolumn{1}{|c|}{ OPCIONES } & FRECUENCIA & PORCENTAJE \\
\hline SI & 44 & $46,32 \%$ \\
\hline NO & 51 & $53,68 \%$ \\
\hline TOTAL & 95 & $100 \%$ \\
\hline
\end{tabular}

Cuadro $N^{\circ}$ 9.- ¿Las evaluaciones de inglés son fáciles de resolver? 
De los 95 estudiantes que realizaron la encuesta el 46,32\% dice que las evaluaciones son fáciles de resolver y el 53,48\% menciona que no; por lo tanto las pruebas de inglés para los estudiantes a menudo son difíciles de resolver demostrando que la procesos de aprendizaje no se están desarrollando adecuadamente y consecuentemente los conocimiento no están siendo bien adquiridos.

\begin{tabular}{|l|c|c|}
\hline \multicolumn{1}{|c|}{ OPCIONES } & FRECUENCIA & PORCENTAJE \\
\hline SI & 80 & $84,21 \%$ \\
\hline NO & 15 & $15,79 \%$ \\
\hline TOTAL & 95 & $100 \%$ \\
\hline
\end{tabular}

Cuadro $N^{\circ} 10 .-_{¿}$ Cree usted que se deben buscar otras maneras de aprender el inglés?

De los 95 estudiantes que realizaron la encuesta el 84,21\% dice que sí se debe aprender inglés de otras maneras a y el 15,79\% menciona que no; en tal virtud se deduce que es necesario la aplicación de nuevas estrategias metodológicas y técnicas de enseñanza aplicadas específicamente a la enseñanza del idioma inglés.

\begin{tabular}{|l|c|c|}
\hline \multicolumn{1}{|c|}{ OPCIONES } & FRECUENCIA & PORCENTAJE \\
\hline SI & 2 & $40 \%$ \\
\hline NO & 3 & $60 \%$ \\
\hline TOTAL & 5 & $100 \%$ \\
\hline
\end{tabular}

Cuadro $N^{\circ} 11 .-$ ¿Utiliza métodos activos para la enseñanza del inglés?

De los 5 profesores encuestados el $40 \%$ dice que sí utiliza métodos activos y el $60 \%$ manifiesta que no, deduciéndose que es urgente realizar una innovación a la metodología vigente, buscando diferentes alternativas de solución que contribuyan al correcto desarrollo de la enseñanza del inglés. 


\begin{tabular}{|l|c|c|}
\hline \multicolumn{1}{|c|}{ OPCIONES } & FRECUENCIA & PORCENTAJE \\
\hline SI & 4 & $80 \%$ \\
\hline NO & 1 & $20 \%$ \\
\hline TOTAL & 5 & $100 \%$ \\
\hline
\end{tabular}

Cuadro $N^{\circ} 12 .-$ ¿Utiliza técnicas variadas para la enseñanza del inglés?

De los 5 encuestados el $80 \%$ afirma que sí utiliza diferentes técnicas para la enseñanza del inglés, y el $20 \%$ dice que no, de esta manera se puede apreciar que es necesario aplicar todas las técnicas de enseñanza que son utilizadas específicamente para la enseñanza de idiomas.

\begin{tabular}{|l|c|c|}
\hline \multicolumn{1}{|c|}{ OPCIONES } & FRECUENCIA & PORCENTAJE \\
\hline SI & 2 & $40 \%$ \\
\hline NO & 3 & $60 \%$ \\
\hline TOTAL & 5 & $100 \%$ \\
\hline
\end{tabular}

Cuadro $N^{\circ} 13 .-$ ¿Enseña inglés a través de juegos, concursos, etc.?

De los 5 profesores encuestados el $40 \%$ de ellos menciona que la enseñanza si lo realiza a través de juegos y concursos, y el $60 \%$ dice que no; de lo que se puede considerar que la enseñanza del inglés a través de juegos, concursos, y demás actividades lúdicas no están siendo aprovechadas eficientemente.

\begin{tabular}{|l|c|c|}
\hline OPCIONES & FRECUENCIA & PORCENTAJE \\
\hline SI & 2 & $40 \%$ \\
\hline NO & 3 & $60 \%$ \\
\hline TOTAL & 5 & $100 \%$ \\
\hline
\end{tabular}

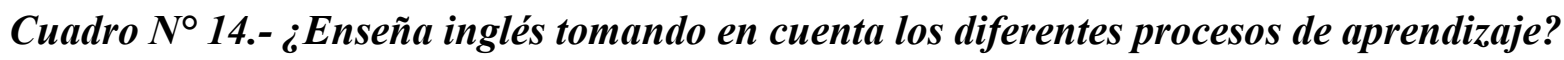

De los 5 profesores encuestados el 40\% manifiesta que sí enseña el inglés tomando en cuenta los procesos de aprendizaje mientras que el $60 \%$ manifiesta que no; por lo que se concluye 
Edison S. Sanguña-Loachamin; Boris E. Vasconez-Torres; Amilcar O. Gonzalez-Diaz; Gabriela M. Moso-Mena

que no se enseña el inglés siguiendo los diferentes procesos de aprendizaje, los mismos que son piezas claves para que el estudiante pueda construir su conocimiento significativamente.

\begin{tabular}{|l|c|c|}
\hline \multicolumn{1}{|c|}{ OPCIONES } & FRECUENCIA & PORCENTAJE \\
\hline SI & 2 & $40 \%$ \\
\hline NO & 3 & $60 \%$ \\
\hline TOTAL & 5 & $100 \%$ \\
\hline
\end{tabular}

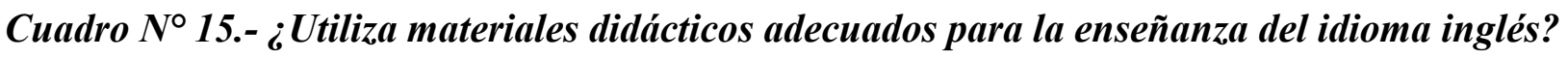

De los 5 profesores encuestados el $40 \%$ manifiesta que si utiliza materiales didácticos específicos para la enseñanza del inglés, por otra parte, el 60\% manifiesta que no; de ello se concluye que, para que el inglés sea bien adquirido por los estudiantes los profesores deben estar bien equipados con toda clase de recursos didácticos apropiados para la enseñanza de idiomas.

\begin{tabular}{|l|c|c|}
\hline \multicolumn{1}{|c|}{ OPCIONES } & FRECUENCIA & PORCENTAJE \\
\hline SI & 1 & $20 \%$ \\
\hline NO & 4 & $80 \%$ \\
\hline TOTAL & 5 & $100 \%$ \\
\hline
\end{tabular}

Caudro $N^{\circ} 16 .-$ ¿Habla con los estudiantes todo el tiempo en inglés?

De los 5 profesores encuestados el 20\% manifiesta que sí hablan todo el tiempo en inglés y el $60 \%$ manifiesta que no; de ello se deduce que los profesores no hablan todo el tiempo en inglés con sus estudiantes, obviando la oportunidad de practicar el idioma de manera real en situaciones de la vida real.

\begin{tabular}{|l|c|c|}
\hline \multicolumn{1}{|c|}{ OPCIONES } & FRECUENCIA & PORCENTAJE \\
\hline SI & 1 & $20 \%$ \\
\hline NO & 4 & $80 \%$ \\
\hline TOTAL & 5 & $100 \%$ \\
\hline
\end{tabular}

Caudro $N^{\circ}$ 17.- ¿Realiza actividades extracurriculares con el fin de mejorar la enseñanza del inglés? 
De los 5 profesores encuestados el 20\% manifiesta que sí realiza actividades extracurriculares mientras que el $60 \%$ manifiesta que no; por lo que se concluye que los profesores deben planificar actividades extra clase como refuerzo a los conocimientos adquiridos de antemano creando espacios propicios para la práctica del idioma.

\begin{tabular}{|l|c|c|}
\hline \multicolumn{1}{|c|}{ OPCIONES } & FRECUENCIA & PORCENTAJE \\
\hline SI & 2 & $40 \%$ \\
\hline NO & 3 & $60 \%$ \\
\hline TOTAL & 5 & $100 \%$ \\
\hline
\end{tabular}

\section{Cuadro $N^{\circ} 18 .-~ ¿ S u s$ estudiantes entienden lo que usted explica en inglés?}

De los 5 profesores encuestados el $40 \%$ manifiesta que los estudiantes sí entienden sus explicaciones y el $60 \%$ manifiesta que no; por lo que se concluye que los profesores necesitan desarrollar actividades tendientes a mejorar la comprensión oral del idioma mediante prácticas orales de repetición y memorización de órdenes y comandos.

\begin{tabular}{|l|c|c|}
\hline \multicolumn{1}{|c|}{ OPCIONES } & FRECUENCIA & PORCENTAJE \\
\hline SI & 2 & $40 \%$ \\
\hline NO & 3 & $60 \%$ \\
\hline TOTAL & 5 & $100 \%$ \\
\hline
\end{tabular}

\section{Cuadro $N^{\circ} 19 .-~ ¿ C r e a$ ambientes propicios para que el estudiante practique el inglés?}

De los 5 profesores encuestados el 40\% manifiesta que sí crean ambientes propicios para la práctica del idioma y el $60 \%$ manifiesta que no; de lo que se desprende que no se brindan las suficientes oportunidades para que los estudiantes practiquen el inglés con actividades relacionadas al medio ambiente y su entorno. 
Edison S. Sanguña-Loachamin; Boris E. Vasconez-Torres; Amilcar O. Gonzalez-Diaz; Gabriela M. Moso-Mena

\begin{tabular}{|l|c|c|}
\hline \multicolumn{1}{|c|}{ OPCIONES } & FRECUENCIA & PORCENTAJE \\
\hline SI & 5 & $100 \%$ \\
\hline NO & 0 & $0 \%$ \\
\hline TOTAL & 5 & $100 \%$ \\
\hline
\end{tabular}

Cuadro $N^{\circ} 20 .-$ ¿Cree usted que se deben buscar alternativas de solución para mejorar el aprendizaje del idioma inglés?

De los 5 profesores encuestados el $100 \%$ manifiesta que si se debe buscar alternativas de solución para mejorar la enseñanza del idioma inglés, por lo que resulta viable la aplicación de estrategias metodológicas activas que ayuden a mejorar la actividad educativa, logrando en los estudiantes la adquisición de aprendizajes significativos.

\begin{tabular}{|l|c|c|}
\hline \multicolumn{1}{|c|}{ OPCIONES } & FRECUENCIA & PORCENTAJE \\
\hline SI & 1 & $75 \%$ \\
\hline NO & 3 & $25 \%$ \\
\hline TOTAL & 4 & $100 \%$ \\
\hline
\end{tabular}

Cuadro $N^{\circ} 21 .-$ ¿Se utiliza metodologías activas para la enseñanza del inglés en su institución?

De las 4 autoridades encuestadas el 25\% manifiesta que si se utilizan metodologías activas en la enseñanza del inglés mientras que el $75 \%$ dice que no; ello demuestra que es necesario innovar la metodología actual y aplicar nuevas estrategias metodologías activas, tendientes a lograr un aprendizaje significativo del idioma inglés.

\begin{tabular}{|l|c|c|}
\hline \multicolumn{1}{|c|}{ OPCIONES } & FRECUENCIA & PORCENTAJE \\
\hline SI & 0 & $0 \%$ \\
\hline NO & 4 & $100 \%$ \\
\hline TOTAL & 4 & $100 \%$ \\
\hline
\end{tabular}

Cuadro $N^{\circ} 22 .-$ ¿La institución cuenta con laboratorios de audio y video específico para la enseñanza del inglés? 
De las 4 autoridades encuestadas el 100\% manifiesta que la institución no cuenta con laboratorios de audio y video dedicados exclusivamente para la enseñanza del inglés; dejando de lado la oportunidad de aplicar las nuevas tecnologías de la información y comunicación, los mismos que aportan significativamente al desarrollo intelectual de los estudiantes.

\begin{tabular}{|l|c|c|}
\hline \multicolumn{1}{|c|}{ OPCIONES } & FRECUENCIA & PORCENTAJE \\
\hline SI & 2 & $50 \%$ \\
\hline NO & 2 & $50 \%$ \\
\hline TOTAL & 4 & $100 \%$ \\
\hline
\end{tabular}

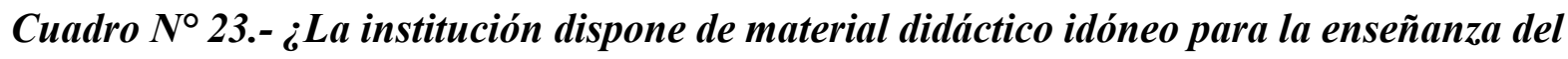
inglés?

De las 4 autoridades encuestadas el 50\% manifiesta que la institución sí dispone de material didáctico idóneo para enseñanza del inglés y el otro 50\% dice que no; haciéndose evidente la necesidad de innovar y equipar continuamente al área de inglés de materiales didácticos especializados y actualizados.

\begin{tabular}{|l|c|c|}
\hline \multicolumn{1}{|c|}{ OPCIONES } & FRECUENCIA & PORCENTAJE \\
\hline SI & 2 & $50 \%$ \\
\hline NO & 2 & $50 \%$ \\
\hline TOTAL & 4 & $100 \%$ \\
\hline
\end{tabular}

Cuadro $N^{\circ} 24 .-~ ¿ S e$ evalúan constantemente los materiales impresos de enseñanza del inglés?

De las 4 autoridades encuestadas el 50\% manifiesta que sí se evalúan los materiales impresos de inglés y el otro $50 \%$ dice que no; ello demuestra que no se realizan evaluaciones periódicas a los diferentes materiales impresos de inglés para su respectiva actualización, innovación equipamiento. 
Edison S. Sanguña-Loachamin; Boris E. Vasconez-Torres; Amilcar O. Gonzalez-Diaz; Gabriela M. Moso-Mena

\begin{tabular}{|l|c|c|}
\hline \multicolumn{1}{|c|}{ OPCIONES } & FRECUENCIA & PORCENTAJE \\
\hline SI & 3 & $750 \%$ \\
\hline NO & 1 & $25 \%$ \\
\hline TOTAL & 4 & $100 \%$ \\
\hline
\end{tabular}

Cuadro $N^{\circ} 25 .-$ ¿Se ejecutan proyectos que generan mejores oportunidades para la adquisición del idioma inglés?

De las 4 autoridades encuestadas el $75 \%$ afirma que si se ejecutan proyectos para mejorar el aprendizaje del inglés y el $25 \%$ dice que no; de lo que se desprende que a los proyectos que están siendo ejecutados en la actualidad hay que reforzarlos con el objeto de mejorar la calidad de la enseñanza de idiomas.

\begin{tabular}{|l|c|c|}
\hline \multicolumn{1}{|c|}{ OPCIONES } & FRECUENCIA & PORCENTAJE \\
\hline SI & 0 & $0 \%$ \\
\hline NO & 4 & $100 \%$ \\
\hline TOTAL & 4 & $100 \%$ \\
\hline
\end{tabular}

Caudro $N^{\circ} 26 .-{ }_{-}$Existen programas de capacitación constante para los profesores de inglés?

De las 4 autoridades encuestadas el 100\% dicen que no existen programas de capacitación constante para los profesores de inglés; de lo que se deduce que los maestros deben estar inmersos en programas de capacitación continua mejorando sus capacidades, destrezas y conocimientos para que la institución pueda contar con profesionales mejor preparados con un alto nivel competitivo.

\begin{tabular}{|l|c|c|}
\hline \multicolumn{1}{|c|}{ OPCIONES } & FRECUENCIA & PORCENTAJE \\
\hline SI & 0 & $0 \%$ \\
\hline NO & 4 & $100 \%$ \\
\hline TOTAL & 4 & $100 \%$ \\
\hline
\end{tabular}

Cuadro $N^{\circ} 27 .-$ ¿Se realizan actividades extracurriculares como: casas abiertas, conferencias, concursos con el fin de mejorar la enseñanza del inglés? 
De las 4 autoridades encuestadas el 100\% dicen que no realizan actividades extracurriculares para mejorar el aprendizaje del inglés; de ello se deduce que la institución debe priorizar la planificación y ejecución de actividades extracurriculares brindando al estudiantado mayores oportunidades de desarrollo de sus capacidades.

\begin{tabular}{|l|c|c|}
\hline \multicolumn{1}{|c|}{ OPCIONES } & FRECUENCIA & PORCENTAJE \\
\hline SI & 0 & $0 \%$ \\
\hline NO & 4 & $100 \%$ \\
\hline TOTAL & 4 & $100 \%$ \\
\hline
\end{tabular}

Cuadro $N^{\circ} 28 .-$ ¿Se brinda oportunidades como: intercambios, becas, pasantías para que los estudiantes puedan tener un contacto directo con el idioma inglés?

De las 4 autoridades encuestadas el $100 \%$ dicen que no existen oportunidades de intercambios estudiantiles; de ello se deduce que urge realizar convenios con instituciones especializadas para que los estudiantes se interrelacionen con personas nativo hablantes mejorando sus oportunidades de aprendizaje.

\begin{tabular}{|l|c|c|}
\hline \multicolumn{1}{|c|}{ OPCIONES } & FRECUENCIA & PORCENTAJE \\
\hline SI & 0 & $0 \%$ \\
\hline NO & 4 & $100 \%$ \\
\hline TOTAL & 4 & $100 \%$ \\
\hline
\end{tabular}

Cuadro $N^{\circ} 29 .-$ ¿Se crea ambientes propicios para que el estudiante practique en todo tiempo el inglés?

De las 4 autoridades encuestadas el 100\% dicen que no se crean ambientes propicios para la práctica del inglés; lo que quiere decir que la institución debe proveer a sus estudiantes todas las oportunidades posibles para la práctica del inglés de manera natural, como lo hacen cuando hablan su lengua materna. 
Edison S. Sanguña-Loachamin; Boris E. Vasconez-Torres; Amilcar O. Gonzalez-Diaz; Gabriela M. Moso-Mena

\begin{tabular}{|l|c|c|}
\hline \multicolumn{1}{|c|}{ OPCIONES } & FRECUENCIA & PORCENTAJE \\
\hline SI & 4 & $100 \%$ \\
\hline NO & 0 & $0 \%$ \\
\hline TOTAL & 4 & $100 \%$ \\
\hline
\end{tabular}

Cuadro $N^{\circ} 30 .-¿$ Cree usted que se debe implementar estrategias metodológicas activas para la enseñanza del idioma inglés?

De las 4 autoridades encuestadas el 100\% dicen que sí se debe implementar estrategias metodológicas activas para la enseñanza del inglés. Ello faculta la aplicación de innovadores estrategias metodologías tendientes a enriquecer el proceso enseñanza aprendizaje del idioma inglés robusteciendo el nivel académico de los estudiantes.

\section{Conclusiones.}

La insuficiente aplicación de métodos activos en la enseñanza del idioma inglés, surge a raíz de la falta de programas de capacitación constante a los profesores del área de inglés de la Unidad Educativa Particular "Cristiano Verbo" ello constituye un factor importante tanto para el desempeño profesional de los maestros, como para el rendimiento académico de los estudiantes, pues de su correcta aplicación dependerá el buen desarrollo de proceso educativo. En este sentido la investigación revela que, a pesar de que un alto porcentaje de encuestados reconoce la importancia de la correcta aplicación de métodos específicos para la enseñanza del inglés, no se observa un interés por cambiar esta realidad.

Por medio de la presente investigación se ha determinado que los estudiantes de la Unidad Educativa Particular "Cristiano Verbo" no tienen las oportunidades suficientes para lograr que el aprendizaje efectivo del idioma inglés, ello acontece en un alto porcentaje debido a que los estudiantes no desarrollan todas sus capacidades, evidenciando que lo que aprenden lo hacen para 
el momento, y como no lo aplican en la vida diaria al poco tiempo se olvidan decir, frente a esta realidad poco se ha hecho por brindarles mejores oportunidades de éxito, facilitándoles todos los recursos para despertar un real interés en la adquisición de una lengua extranjera. Debido a la mala experiencia adquirida en el proceso de enseñanza-aprendizaje, los niños y jóvenes estudiantes sienten temor y tratan de aprender el inglés bajo la presión de un pensum académico mal diseñado, la presión de cumplir con la aprobación de la asignatura, y en ciertos casos los estudiantes tratan de aprender solo por obligación y no por iniciativa propia perdiendo el interés y motivación por aprender naciendo un rechazo hacia el idioma.

La mayoría de los estudiantes de la Unidad Educativa Particular "Cristiano Verbo" manifiestan que las estrategias metodológicas influyen en el aprendizaje significativo del idioma inglés, por lo tanto consideran que el desarrollo de las clases puede mejorar si se perfeccionan dichas estrategias, las mismas que deben ser aplicadas de acuerdo a su realidad y respetando los diferentes procesos de aprendizaje. . Los maestros encuestados consideran el rendimiento académico de los estudiantes tienen limitaciones, no desarrollan toda su creatividad, sus habilidades y sus destrezas ello se evidencia en la poca participación de los estudiantes en clase y más aún cuando tienen que expresar sus ideas en un idioma extranjero. El siguiente obstáculo que se presenta es que los estudiantes no están preparados totalmente para adquirir dicho idioma de una manera práctica y efectiva, esto se debe entre otras a que las instituciones educativas no cuentan con todos los recursos suficientes e idóneos que les brinde la oportunidad de desenvolverse en un ambiente de aprendizaje significativo del idioma inglés. 


\section{Recomendaciones.}

Es imprescindible la actualización aplicación de métodos activos para la enseñanza del idioma inglés. La Unidad Educativa Particular "Cristiano Verbo" debe ampliar los programas de capacitación docente orientados a la actualización de sus conocimientos y a perfeccionamiento de sus habilidades, dotándoles de todas las herramientas y recursos pedagógicos suficientes para que cada profesor de inglés pueda desarrollar sus clases con motivación, mejorando su rendimiento laboral.

El aprendizaje significativo se da cuando el estudiante relaciona sus conocimientos previos con los nuevos para de esta manera retenerlos y ponerlos en práctica de forma natural en situaciones reales, por ello los estudiantes deben tener a su disposición todos los recursos necesarios para su correcto desarrollo estudiantil, la institución, los profesores, y el sistema educativo debe solventar las necesidades pedagógicas y de infraestructura, creando motivación y aumentando el interés por aprender un idioma que le permitirá ampliar sus horizontes en el ámbito profesional.

Una guía de métodos específicos para la enseñanza del idioma inglés proporcionará a los profesores una gama de innovadoras alternativas para llevar a cabo su labor docente de una manera eficiente, creando momentos adecuados e interesantes, creando motivación y mayores oportunidades de éxito en el aprendizaje del inglés, todo esto desarrollado en un ambiente propicio, pertinente y competitivo.

\section{Bibliografia.}

Brown, D. (2000). Principles of language learning and teaching. N.Y. USA: Longman.

Duarte de Kendler, M. (2007). Impacto de las metodologías de enseñanza utilizadas por el docente sobre la efectividad del aprendizaje del idioma inglés. Telos, 9(2), 301-317. 
González, M. (2000). Lúdicas y Lenguas Extranjeras. Cómo desarrollar competencias comunicativas. Santafé de Bogotá: Cooperativa Editorial Magisterio.

Lightbown, P., \& Spada, N. (1996). How Languages are Learned. New York: Oxford University Press.

Richards, \& Rodgers . (2001). Approaches and Methods in Language Teaching (2 ed.). USA: Cambridge UniversityPress. 